\title{
Masking by light and the sustained-transient dichotomy
}

\author{
MARC GREEN \\ Indiana University, Bloomington, Indiana
}

\begin{abstract}
An interpretation of masking by light in terms of the sustained-transient dichotomy is presented. It is proposed that presentation of a uniform flash has two effects: a masking effect, which occurs within the transient system, and a facilitation effect due to increased adaptation level. These two components have been separated by the employment of uniform flashes (flicker around a mean luminance) that do not change adaptation level. Results of such studies generally suggest that masking by light occurs only when targets are detected by the transient system. However, not all results are consistent with the sustained-transient model. Reexamination of other recent literature reveals that the motion/pattern dichotomy underlying the model is not tenable. Moreover, other evidence appears to refute the view that the sustained system dominates fovea vision while the transient system is more responsive in the periphery. I sug. gest a modified version of the dichotomy and propose a simple receptive field model of sustained and transient mechanisms.
\end{abstract}

This article is written with three goals in mind. The first is to propose a new interpretation, based on the sustained-transient model, of the masking-by-light phenomenon. The second is to reexamine the validity of the sustained-transient dichotomy and the criteria on which the dichotomy is based. And the third is to present a receptive field model which seems to account for most of the differences between sustained and transient mechanisms.

The sustained-transient model (Breitmeyer \& Ganz, 1976; Keesey, 1972; Kulikowski \& Tolhurst, 1973; Tolhurst, 1973) has become one of the most influential theories in vision. It has been used to explain a number of phenomena, including metacontrast masking (Breitmeyer \& Ganz, 1976) and pattern masking (Legge, 1978; Pantle, 1983). In this paper, I will attempt to show that the model can be used to largely explain masking by light. Most of the evidence for my interpretation comes from the studies I have conducted, although I will also include converging evidence from other sources.

My analysis derives largely from studies in which observers view sinusoidal gratings in the presence of uniform flicker. Data generated by this paradigm were generally consistent with an explanation of masking based on the sustained-transient dichotomy. As the studies progressed, however, it became clear that not all of the results could be reconciled with the most common versions of the sustained-transient model. Moreover, a review of other data published

This research was partially supported by NIH Grant R01 EY 5292-01. The author's mailing address is: Visual Sciences Department, Indiana University, 800 East Atwater Street, Bloomington, IN 47405. in the period since the model was originally proposed reveals several findings that are inconsistent with some of the dichotomy's basic tenets. Although the validity of the dichotomy itself has been disputed (e.g., Burbeck, 1981; Derrington \& Henning, 1981; Lennie, 1980a, 1980b), I believe, in light of more recent data, that a model with separate sustained and transient systems can still be retained if some modifications and updating are performed. In sum, it appears that the human visual system cannot be rigidly divided into pattern (sustained) and flicker/motion (transient) analyzing systems with different spatiotemporal properties. The view that the two mechanisms have different relative sensitivities in central and peripheral vision also appears insupportable. However, several criteria still allow two mechanisms to be distinguished. The differences can largely be explained by a receptive field model that takes into account variations in the spatial and temporal distributions of inhibition.

The first part of this paper will briefly describe the sustained-transient model of human vision. A second section will outline the series of experiments that led me to question the sustained-transient distinction as presented by most authors. I will propose an account of masking by light in terms of the dichotomy and suggest that the basic nature of masking by light has been misunderstood. The subsequent section will present a modified version of the dichotomy and will cite supporting evidence from additional studies. Finally, I will suggest a simple receptive field model which seems to account for most of the differences between sustained and transient mechanisms.

Before beginning, it should be made clear that this paper is concerned only with psychophysical mechanisms. The terms "sustained," "transient," and 
"receptive field" refer only to the inferred perceptual mechanisms and not to physiological ones. The early basis of the dichotomy was derived primarily from single-unit recording studies (e.g., Enroth-Cugall \& Robson, 1966) in which cells were classified into various categories by some response criterion. However, the sustained-transient distinction is something like Frankenstein's monster: it may have been created by physiologists, but it now has a life of its own. While support for the physiological basis of the model has eroded somewhat (Lennie, 1980a, 1980b), there has been much psychophysical evidence which purports to demonstrate the dichotomy's validity. Therefore, I will restrict the discussion in this article to inferred psychophysical mechanisms. Any connection to single units will be left to the reader's imagination.

\section{THE SUSTAINED-TRANSIENT DICHOTOMY}

The notion that the brain might contain separate mechanisms for encoding stationary as opposed to moving objects was first proposed by Bowditch and Hall (1881). Observers who had adapted to an expanding spiral perceived a "chaotic" motion aftereffect when they subsequently closed their eyes. If a stationary stripe was laid across the spiral at the end of adaptation, the observers, after closing their eyes, saw the motion aftereffect superimposed on the stationary afterimage of the stripe. (See Green, Chilcoat, \& Stromeyer, 1983, for a similar finding.) Bowditch and Hall then surmised the existence of different "cerebral centers" for motion and "rest." More recently, Nes, van Koenderink, Nas, and Bouman (1967) noted that when observers viewed a drifting grating, separate thresholds could be determined for the perception of flicker, motion, and pattern. Keesey (1972) is usually credited as the first to propose that the different threshold criteria may represent separate pattern and flicker/motion perceptual systems. At threshold, a temporally modulated line was perceived as a diffuse flicker. Observers could then determine a second, higher threshold for seeing well-defined form. These thresholds were interpreted as defining the sensitivities of two separate retinal detection mechanisms. The sustained-transient distinction was subsequently reinforced in studies by Tolhurst (1973) and Kulikowski and Tolhurst (1973) and then popularized by the well-known Breitmeyer and Ganz (1976) article. These papers established what I will term the "original" version of the sustained-transient distinction.

According to the original version, the two mechanisms differ in several respects. First, the sustained and transient systems are presumed to have different spatiotemporal tunings. The sustained system is optimally sensitive to stationary gratings of moderate spatial frequency. That is, it is low pass in the temporal frequency domain but bandpass in the spatial frequency domain (Figure 1). The transient system,
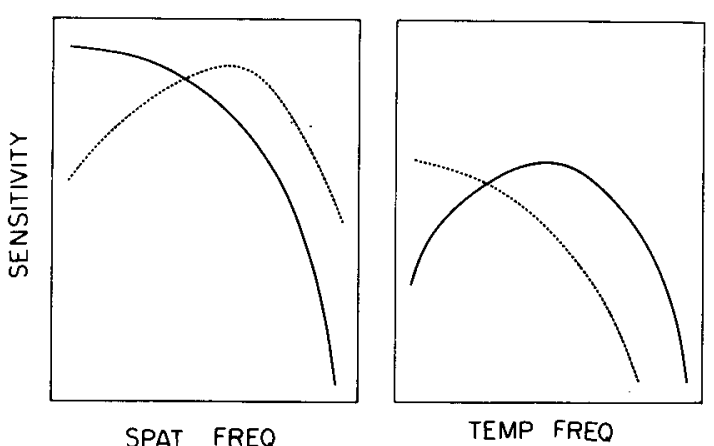

TEMP FREO

Figare 1. Hypothetical sensitivities of sustained and transient mechanisms. Left panel shows sensitivity for sustained (dashed line) and transient systems (solid lines) as a function of spatial frequency. Right panel represents sensitivities as a function of temporal frequency.

on the other hand, is tuned to low spatial frequencies (low pass spatially) and moderate temporal frequencies (bandpass temporally). In addition to sensitivity differences, the mechanisms differ functionally. The sustained system signals the presence of stationary spatial pattern, whereas activation of the transient system results in the perception of temporal change (flicker or motion) but no well-defined spatial structure. Some investigators (Burbeck, 1981; Derrington \& Henning, 1981), however, have misinterpreted previous authors, claiming they had suggested that the transient system could signal no spatial variation in luminance. To my knowledge, no proponent of the distinction has ever asserted such an extreme position. On the other hand, extreme statements have been made with regard to the motion-processing ability of the sustained system. It is usually claimed that the sustained system can signal no information about motion or flicker (Breitmeyer \& Ganz, 1976; Kulikowski \& Tolhurst, 1973), that it can signal information only about a stationary pattern. This assertion is critical to the original version of the dichotomy because the most common method of investigating the mechanisms is the determination of separate pattern and motion/flicker thresholds. Following Keesey (1972), the presumption is made that pattern thresholds represent the lower bound of the sustained system, whereas flicker/motion thresholds indicate the threshold sensitivity of the transient system. This logic has been often used to reveal the spatial frequency tunings of the two mechanisms (e.g., Levi \& Harwerth, 1977).

The prototypical experiment is conducted in the following manner. An observer views drifting gratings and sets threshold using pattern as well as motion/ flicker criteria. At high spatial frequencies (see Figure 1, right panel), the sustained system is more sensitive so that the observer first sees stationary stripes. In order for motion/flicker to be seen, contrast must then be increased to activate the less sensitive transient system. When low spatial frequencies are viewed, 
the transient system is more sensitive. The observer initially perceives motion without clearly defined stripes. The spatial structure can be recognized only after contrast is increased sufficiently to reach threshold of the sustained system.

In addition to the primary characteristics noted above, the sustained and transient mechanisms are presumed to differ in other respects. The transient system is said to respond with a shorter latency (Breitmeyer, 1975; Lupp, Hauske, \& Wolf, 1976; Vassilev \& Mitrov, 1976) and a briefer temporal summation (Breitmeyer \& Ganz, 1977; Brown \& Black, 1976; Legge, 1978). Several authors (Breitmeyer \& Ganz, 1976; Sharpe, 1974) have further proposed that the transient system is relatively more sensitive in the periphery, whereas the sustained system dominates in the central visual field. Finally, it has been claimed that the sustained system exhibits a retinal anisotropy, but that the transient system exhibits no oblique effect (Camisa, Blake, \& Lema, 1977) or only a reduced one (Essock \& Lehmkuhle, 1982). These studies reported that pattern thresholds were higher for tilted than for vertical gratings, but that orientation had little or no effect on flicker thresholds.

Now that the properties of the two mechanisms have been outlined, I will describe the series of experiments performed in order to apply the model to masking by light. Data from these experiments raise questions about the validity of many distinctions previously used to define the sustained-transient dichotomy.

\section{MASKING BY LIGHT}

Kahneman (1968) has suggested that there are at least two distinct classes of masking. In "masking by light," the mask is a uniform field that contains no spatial variations in luminance (except for the edges of field); "masking by pattern" occurs when the mask contains internal contours. Although the studies reported below fall into the category of masking by light, Legge's (1978) masking-by-pattern study of the sustained-transient dichotomy found results that dovetail nicely with light-masking data. Legge's study will be discussed below.

\section{Crawford Masking of Gratings}

The first major masking-by-light study was performed by Crawford (1947). Observers detected a 0.5-deg-diam spot of light presented briefly against a larger, spatially uniform conditioning field. The test spot was flashed with various asynchronies before, during, or after a 524-msec mask. Results of the study, shown schematically in Figure 2a, exhibited three main features: (1) threshold rose even when the test flash was presented before the mask (backward masking), (2) the higher the conditioning field luminance, the greater the masking, and (3) threshold
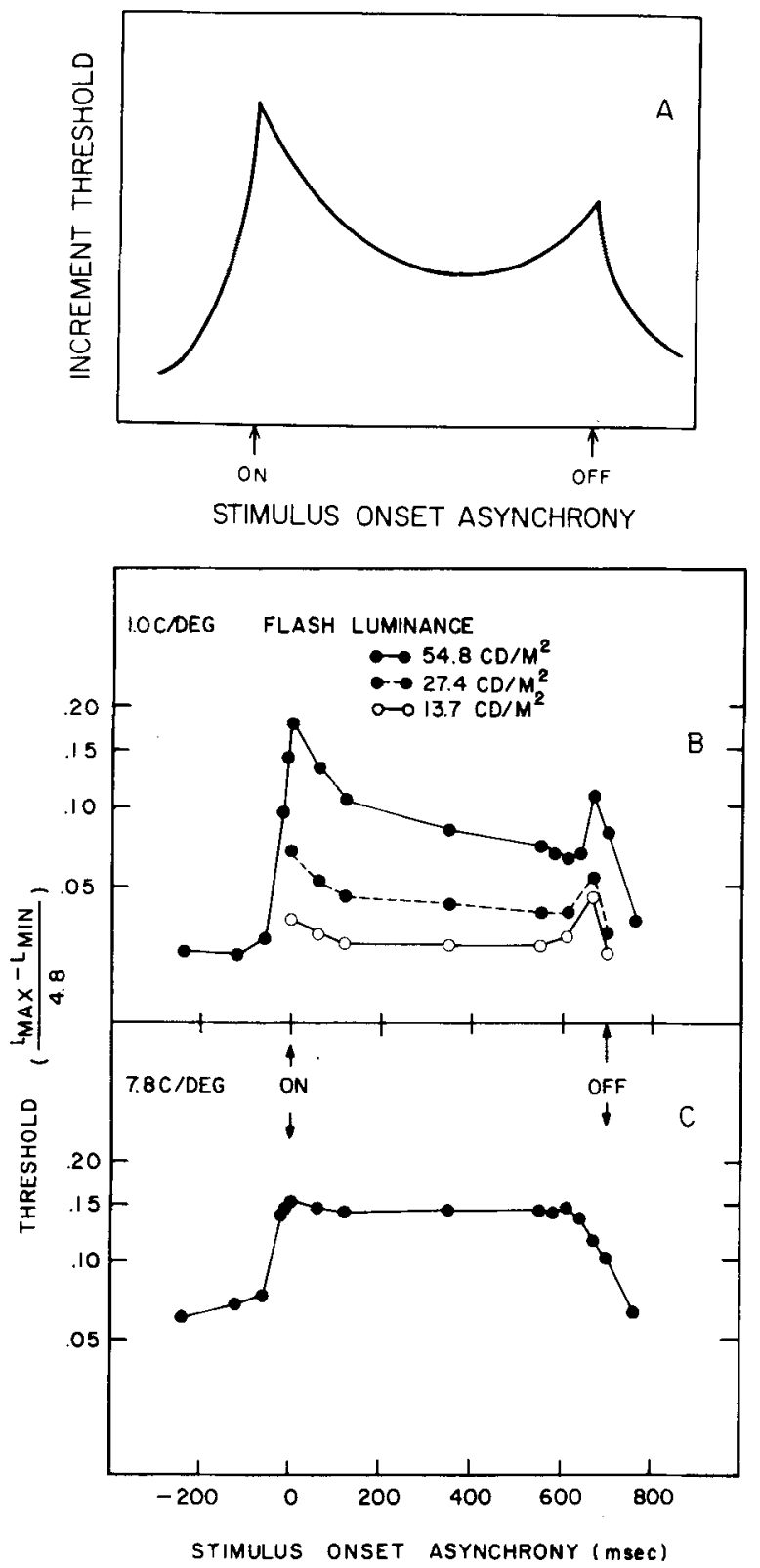

Figure 2. Date obtalned from Crawford madking paradigm. Panel A schematically shows inerement thresholds obtained by Crawford (1947) when a 0.5-des test spot was maked with a 524msec light flash. Panel B shows increment thresholds for detection of a 1.0-c/deg grating masked by a 700 -msec uniform flash. Panel C shows results obtained with a 7.8-c/des grating (Green, 1981a).

is highest at mask on and offset, producing "ears" in the masking function. An interpretation of this effect made by Sperling (1965) was strikingly similar to the subsequent sustained-transient notion. He proposed that masking by light actually consisted of two components, one transient and the other steady state. Observers were presumed to normally detect the test spot by the "temporal pattern of illumination." 
When the test spot was presented at the beginning or end of the mask, however, the mask's "impulse response" transiently decreased sensitivity for the temporal pattern. Observers were then forced to use "spatial contrast," which was less efficient. When presented in the middle of the mask, the observers could return to using the more detectable temporal pattern so that threshold decreased. Sperling's suggestion has often (e.g., Kahneman, 1968) been interpreted as meaning that the observer shifts his decision criterion. However, another possible inference is that detection of temporal pattern and spatial contrast is mediated by different visual mechanisms. The second component of masking was a steady state change of sensitivity due to increased light adaptation.

In view of the sustained-transient dichotomy, Sperling's hypothesis suggests that masking by light would depend greatly on the spatial frequency content of the target. The test spots used by Crawford and most subsequent experimenters contain its highamplitude components at low spatial frequencies. When flashed briefly, the spots should, because of the transient system's greater sensitivity-at low spatial frequencies and its shorter temporal summation period, be detected as "temporal impulses." On the other hand, detection of a high spatial frequency target would be mediated by the sustained system since the observer would always rely on spatial contrast. Masking curves for a high spatial frequency grating should exhibit no ears. Figures $2 \mathrm{~b}$ and $2 \mathrm{c}$ show the results for an experiment (Green, 1981a) in which the observer adjusted contrast to detect a grating of either 1.0 or $7.8 \mathrm{c} / \mathrm{deg}$ presented on a CRT screen for $30 \mathrm{msec}$ before, during, or after a 700-msec conditioning flash. As predicted by Sperling's hypothesis (and the sustained-transient model), the low spatial frequency grating produced ears, whereas the high spatial frequency grating did not. This presumably occurred because the high spatial frequency grating was always detected by spatial contrast, rendering the conditioning flash transients ineffective in masking. Kitterle and Leguire (1975) have likewise found ears at mask offset when observers detected spots but not fine gratings.

This seemed to support the sustained-transient distinction in masking by light, but one problem remained. Although there were no ears, threshold for detecting the high spatial frequency grating was elevated by the mask. If the uniform flash selectively masked the transient system, why was threshold for detection of the high spatial frequency elevated? The answer lies in the difference between the use of increment threshold and contrast threshold as dependent measures. The increment threshold measure used by Crawford indexes the absolute amount of light needed to detect the target, whereas contrast sensitivity indicates the amount of light relative to the background. The data shown in Figure 2b are analogous to incre- ment thresholds, since I plotted only the contrast of the grating on the CRT without adding in the extra light from the conditioning flash (LCF). When this is included (Figure 3), the effect of the conditioning flash appears quite different: contrast sensitivity for detecting the grating actually increases. This should be no surprise, inasmuch as several studies (e.g., Patel, 1966) have reported that grating sensitivity improves with higher mean luminance. Since the visual system seems designed to detect contrast rather than absolute light levels (Barlow, 1972), it appears fair to say that "masking" by light is something of a misnomer. Sensitivity to the high spatial frequency grating is uniformly increased by the "mask." With low spatial frequency gratings, there appears to be a genuine masking effect at the on- and offset of the background flash, but this is superimposed on the improvement due to the higher mean luminance.

The explanation of masking by light here proposed leads to an interesting possibility: presentation of uniform flashes without a change in mean luminance will produce only the transient component of masking. To demonstrate this, I have conducted several experiments in which observers have detected gratings in the presence of two kinds of background. The first background consisted of a uniform field with a steady mean luminance. The other was a uniform field that sinusoidally flickered about the same

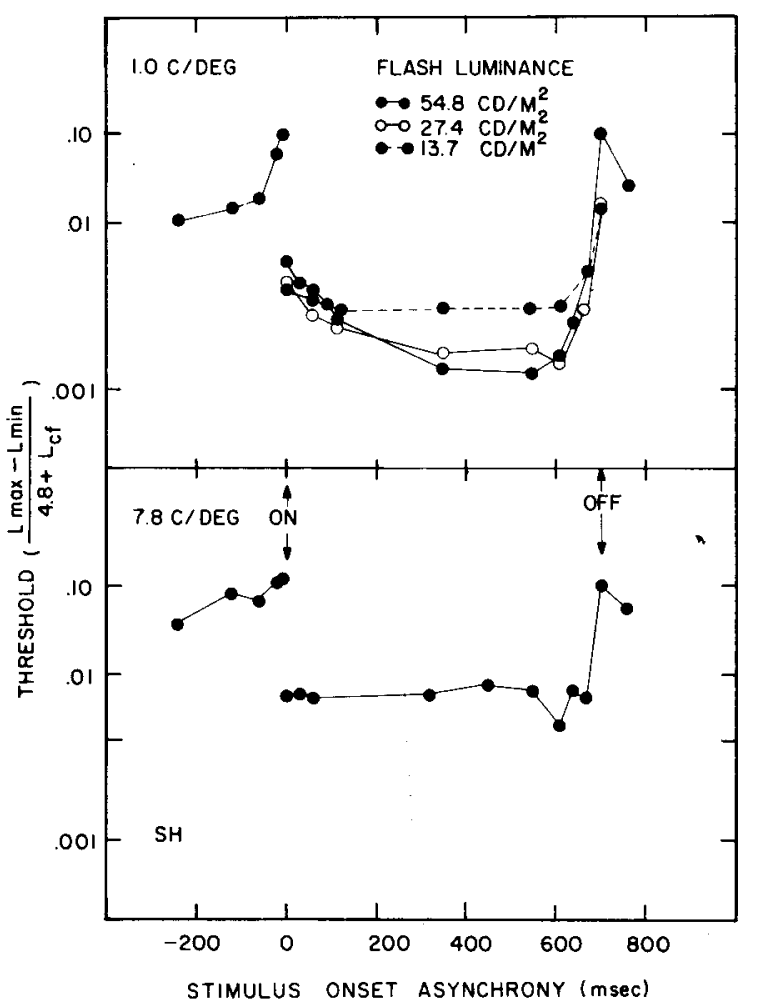

Figure 3. Contrast threshold for 1.0- and 7.8-c/deg gratings masked by a 700-msec light flash (Green, 1981a). 
mean luminance. Time-averaged luminances of the flickering and steady fields were identical so that they produced the same level of light adaptation, but the flickering light contained transients.

\section{Interocular Transfer}

For further evidence of separate transient and luminance components to visual masking, Green and Odom (1984) measured interocular transfer of flicker masking. It is often stated (e.g., Boynton, 1961; Kahneman, 1968) that there is little or no interocular transfer of masking by light. Actually, this is not quite true. Dichoptic effects can be quite large, as evidenced by the $0.5 \mathrm{log}$ unit effect reported by Wagman and Battersby (1959). However, the dichoptic effect is usually small compared with the magnitude of monoptic masking. The difference might be that light adaptation effects are primarily monocular (Levelt, 1968). Therefore, both of the components are involved in the monoptic situation, but only transient masking, which appears to have a smaller effect, transfers interocularly. With uniform flicker, however, there is no steady luminance effect, so monoptic and dichoptic transient masking can be compared directly. Observers detected a drifting grating $(0.67$ c/deg at $8 \mathrm{~Hz}$ ) with one eye while flicker was presented to the same or contralateral eye. Dichoptic masking proved only $0.2 \log$ unit weaker than monoptic masking. Much larger monoptic-dichoptic differences have been reported in studies using a steady-state luminance component. Wagman and Battersby (1959) found a $2.5 \mathrm{log}$ unit monocular superiority, and Boynton (1961) showed differences of 0.83 and $1.6 \mathrm{log}$ units in two different experiments.

Some studies have failed to find any dichoptic masking by light. For example, Schiller (1965) determined thresholds for identifying alphabetic characters followed by a uniform flash. The mask increased thresholds in monoptic conditions but not when stimulation was dichoptic. The letter-identification task, however, is a test of pattern discrimination, which is presumably mediated by the sustained system. On the other hand, detection of flashed spots and lowfrequency gratings is performed by the transient system. This disparity in results with different targets reinforces the view that masking involves separate light-adaptation and transient components and that only the transient component transfers interocularly.

\section{Adaptation to Flicker}

Since they contain the transient component and not the steady-state component, flicker masks should cause a loss of sensitivity for detecting low spatial frequency gratings but have no effect on seeing high spatial frequency ones. In another experiment (Green, 1981b), this hypothesis was tested by determining the effect of prior flicker adaptation on the sensitiv- ity for drifting gratings. (For technical reasons, adaptation was used rather than masking. As will be shown below, however, the two paradigms give identical results.) Neither prior viewing of flicker nor masking by flicker affected detection thresholds for stationary gratings of any spatial frequency (see also Klein, Stromeyer, \& Madsen, 1977). This is, of course, consistent with the assertion that stationary patterns are detected by the sustained system, whereas the mask is processed by the transient system. When the test gratings were flickered, exposure to uniform flashes produced a sensitivity loss at low spatial frequencies (Figure 4a). As spatial frequency increased, the magnitude of the effect decreased and was totally absent at about 2-4 c/deg. ${ }^{1}$ The data shown here were obtained with a $16-\mathrm{Hz}$ rate, but similar effects occurred at $8 \mathrm{~Hz}$. The spatial frequency tuning of the effect is consistent with the sustained-transient dichotomy, which presumes that the transient system mediates detection only at low spatial frequencies.

On the surface, this experiment appears to support the original version of the sustained-transient dichotomy. However, there was one troubling aspect to the study. Observers noted that gratings of all spatial frequencies appeared to move both before and after flicker adaptation. Apparently, uniform flicker had no effect on detection of high spatial frequency gratings, even though their motion was clearly perceptible. This suggested that uniform flicker would not always impair motion perception.

To make this observation explicit, a subsequent experiment was performed in which observers were asked to adjust threshold based on one of two criteria. The "detection" criterion was defined as the perception of any spatial or temporal inhomogeneity on the screen. Observers were to state, however, whether the test grating appeared stationary (S) or temporally modulated $(T)$ at threshold. If stationary, the observers were then to adopt the "transient" criterion and increase contrast until motion/flicker could be seen. The simple prediction, based on the sustainedtransient model, was that flicker should raise threshold only if the grating had appeared temporally modulated (T) prior to adaptation. Results obtained with 8-Hz on-off flicker are shown in Figure 5. As is usually found, low spatial frequency gratings appeared to be temporally modulated, whereas the finer gratings were seen as stationary. The effect of flicker was to raise threshold for gratings that appeared prior to adaptation to be temporally modulated, that is, gratings as fine as $1.5 \mathrm{c} / \mathrm{deg}$. Thus, part of the prediction was confirmed. The lower panel shows the results obtained when a transient criterion was adopted. Flicker adaptation effects were extended to spatial frequencies as high as $4 \mathrm{c} / \mathrm{deg}$. Even though the motion/flicker criterion was employed, the uniform flicker had no effect on thresholds above $4 \mathrm{c} / \mathrm{deg}$. 

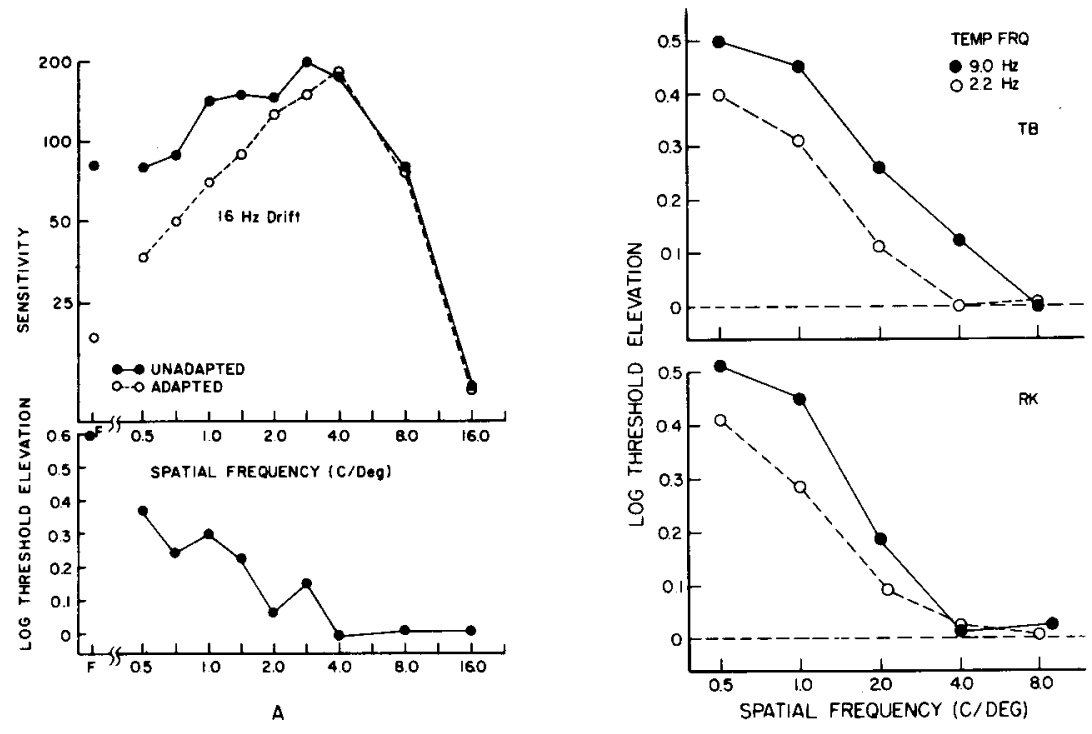

B
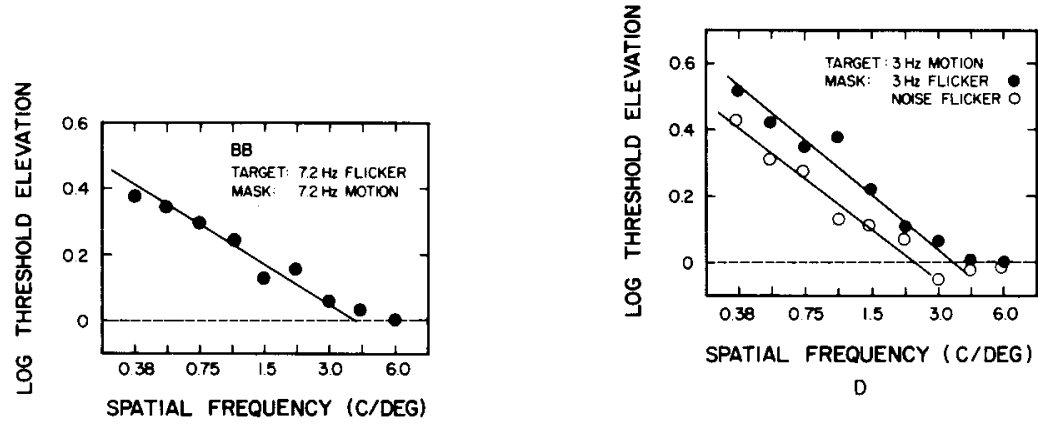

SPATIAL FREOUENCY (C/DEG)

D

$C$

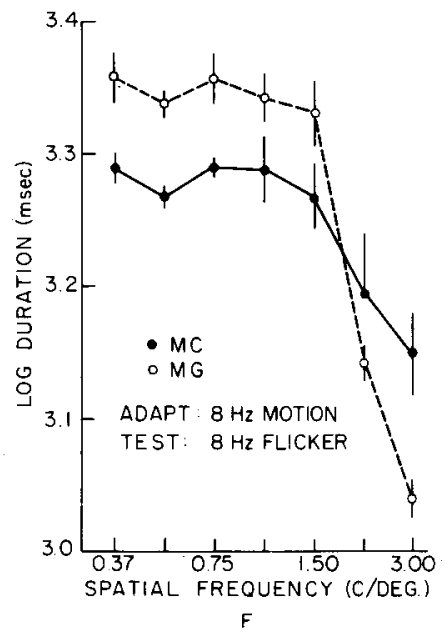

Figure 4. The effect of uniform field flicker on moving gratings of different spatial frequencies. (A) Observer adapts to 16-Hz flicker and detects gratings drifting at $16 \mathrm{~Hz}$ (Green, 1981b). (B) Observer detects drifting gratings masked by simultaneously presented flicker (Green, 1983b). (C) Observer detects flicker masked by drifting gratings (Green, 1983c). (D) Observer detects drifting gratings masked by sinusoidal or noise flicker (Green, 1983c). (E) Observer detects drifting gratings masked by either 3- or 12-deg-wide bars (Green, 1983a). (F) Duration of motion aftereffect seen in uniform flicker following adaptation to gratings of various spatial frequencies (Green, Chilcoat, \& Stromeyer, 1983). 


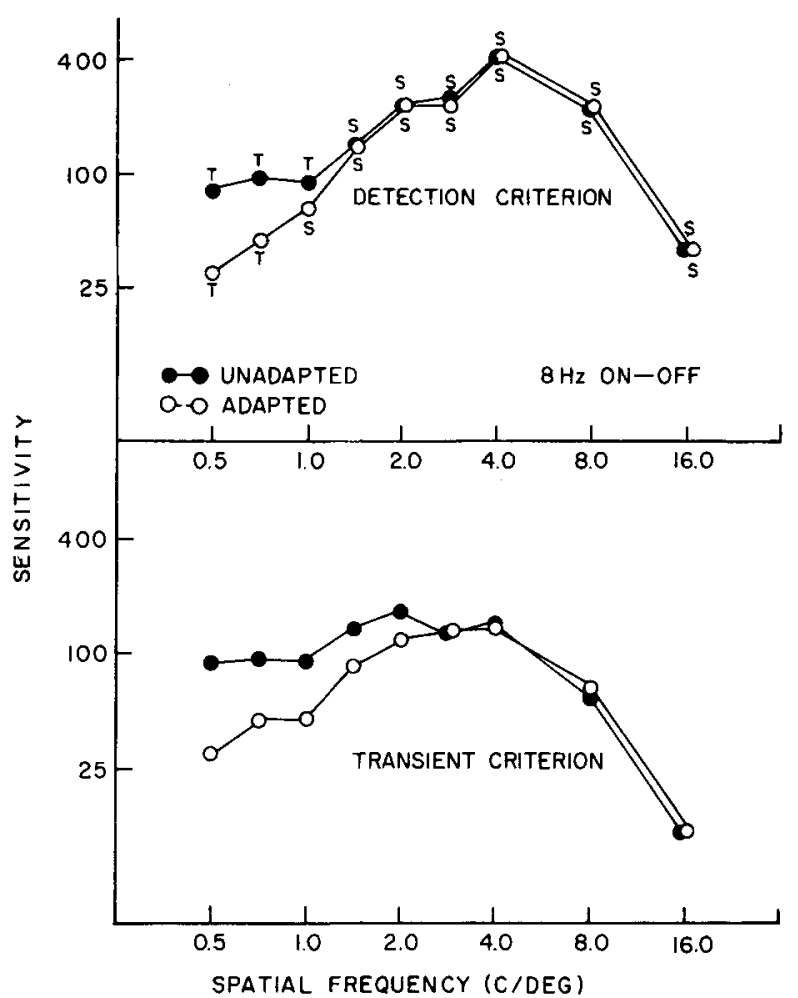

Figure 5. Contrast thresholds for detection of drifting gratings by two criteria. Top panel shows data obtained with the "detection" criterion, which consisted of any deviation from a uniform field. Lower panel shows "translent" criterion results obtained when the criterion was perception of temporal change (Green, 1981b).

This is certainly not consistent with the view that the sustained-transient difference is one of form vs. motion perception. Note also that the transient system cuts off at $4 \mathrm{c} / \mathrm{deg}$. Other authors (e.g., Breitmeyer \& Ganz, 1976; Kulikowski \& Tolhurst, 1973) have suggested that the transient system operates well above $4 \mathrm{c} / \mathrm{deg}$, although it may be less sensitive than the sustained system. I have found no evidence of transient mechanisms above $4 \mathrm{c} / \mathrm{deg}$ (see below).

\section{Flicker Masking}

Possible problems with the use of subjective pattern and movement criteria have been often pointed out (e.g., Burbeck, 1981). The aim of the next experiment (Green, 1983b) was to determine the effect of flicker on grating detection in a more rigorous fashion. In addition, I wished to validate the prediction that uniform flashes presented without change in mean luminance would have no effect on the detection of high spatial frequency gratings. This experiment involved the use of a detection-discrimination paradigm in which observers were tested in a series of two-alternative forced-choice trials. A grating (drifting at 2.2 or $9.0 \mathrm{~Hz}$ ) was presented in one of the two intervals, with direction of motion varying from trial to trial. In detection trials, the observer indicated which of the two intervals contained the test grating. During a separate set of trials, the task was to discriminate direction of motion, and no response as to interval was required. Thresholds for detection and discrimination were then compared. If thresholds were similar, then it was assumed that the mechanism responsible for detection was also a motion analyzer (i.e., a transient mechanism). If more contrast was required for discrimination because the grating appeared stationary at threshold, then it was assumed that detection was mediated by a pattern (sustained) mechanism. Interpretation of masking in terms of the sustained-transient dichotomy then suggests the following predictions: (1) If detection and discrimination thresholds are similar, then processing was mediated by the transient system and masking should occur; and (2) if discrimination threshold is higher than detection threshold, then detection was mediated by the sustained system and no masking should occur. At a drift rate of $2.2 \mathrm{~Hz}$, detection and discrimination thresholds are similar for spatial frequencies of $0.5,1.0$, and $2.0 \mathrm{c} / \mathrm{deg}$ but unequal at 4.0 and $8.0 \mathrm{c} / \mathrm{deg}$. At a drift rate of $9.0 \mathrm{~Hz}$, motion could be discriminated at threshold for all spatial frequencies. The data, shown in Figure $4 \mathrm{~b}$, partly confirm the predictions. Uniform flashes presented without change in mean luminance impair detection at low spatial frequencies but have no effect on sensitivity at high spatial frequencies. Similar results were obtained when drifting gratings of different spatial frequency were used to mask flicker (Green, 1983c) (Figure 4c) and when the mask was broadband noise flicker rather than sinusoidal flicker (Figure 4d). To this extent, the masking data are consistent with the sustained-transient model. A closer examination also reveals that there is good agreement between data

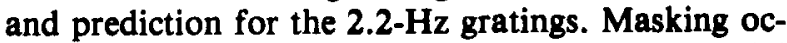
curs only for gratings as fine as $2.0 \mathrm{c} / \mathrm{deg}$, the spatial frequency range in which motion was discriminated at threshold. However, predictions were not entirely confirmed at higher drift rates. At $9.0 \mathrm{~Hz}$, no masking occurred at $8.0 \mathrm{c} / \mathrm{deg}$ for both observers or at $4.0 \mathrm{c} / \mathrm{deg}$ for one, even though motion could be discriminated at threshold. These data strengthen the conclusion that masking by light does not affect detection of high spatial frequency gratings, even when they appear to move.

\section{Ruling Out Other Explanations}

The results of the experiments described above can be summarized as follows: (1) Uniform flashes of light produce a transient masking effect in targets of low but not high spatial frequency (above $4 \mathrm{c} / \mathrm{deg}$ ), and (2) the targets must appear to move or be briefly flashed, because no masking occurred with steadily presented gratings. In general, masking by light is readily understandable in terms of the transient- 
sustained dichotomy. Since uniform flicker is presumably processed only by the transient system (Wilson, 1980), masking will be found only when the test target is also transmitted by the transient system. A dissonant aspect to the data is that no masking occurs above $4 \mathrm{c} / \mathrm{deg}$, even though the gratings may appear to move. Two conclusions seem possible. First, motion at high spatial frequencies is not processed by the transient system. This would mean that sustained and transient mechanisms cannot be distinguished on the basis of motion perception. Second, uniform flicker desensitizes only those transient mechanisms tuned to low spatial frequency.

The second possibility was evaluated by some supplementary experiments. One reason that flicker might affect sensitivity only to coarse gratings is temporal tuning. When spatial frequency was increased, temporal frequency was held constant. Since velocity is equal to temporal frequency divided by spatial frequency, increasing spatial frequency resulted in a proportional decrease of velocity. Perhaps what appeared to be spatial frequency tuning was really velocity tuning. An experiment (Green, 1983b) was therefore conducted in which $2.2-\mathrm{Hz}$ flicker was used to mask gratings of different spatial frequencies but with the same velocity of $4.4 \mathrm{deg} / \mathrm{sec}$. The masking curve that resulted was virtually identical to those obtained with fixed temporal frequency. The spatial frequency range of mask does not seem to depend on velocity. Moreover, masking has the same 2-4c/deg upper limit when temporal frequency was as high as $16 \mathrm{~Hz}$ (Green, 1981b) or as low as $2.2 \mathrm{~Hz}$ (Green, 1983b).

Another reason that threshold elevations may occur only with coarse gratings is the spatial frequency tuning of masking. The uniform flickering field contains edges that produce low spatial frequency components in the display. Since masking is strongest when target and mask contain the same components (e.g., Stromeyer \& Julesz, 1972), this could explain the spatial frequency tuning that I obtained. To test this possibility, the dimensions of the mask were varied independently from those of the target. Figure 6 schematically shows the visual display. The test grating was multiplied by a cosine function to produce a 3-deg-wide patch. Flicker covered rectangular areas 3 or 12 deg wide, so that the spectral components of the two masks differed by a factor of 4. A spatial frequency-specific masking effect would be indicated if the 3-deg-wide flicker masked gratings 4 times higher in frequency than those masked by the 12-deg-wide flicker. The results, shown in Figure 6c, were virtually identical for the two masks (Green, 1983b). Threshold elevation again decreased from a maximum at the lowest spatial frequency to nil at $4 \mathrm{c} / \mathrm{deg}$. In other versions of this experiment, the flicker mask consisted of a 3-deg-wide and 11-deghigh rectangle (that is, the flicker bar was rotated

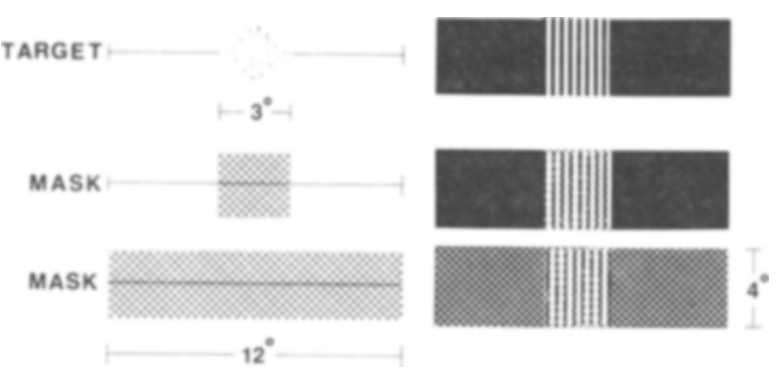

Figure 6. Schematic of the stimuli used to determine whether size of the mask affects flicker masking. Hatched areas represent location of the uniform flicker. Square-wave gratings are shown, but sinusoidal patterns were used in the actual experiment.

$90 \mathrm{deg}$ ) or an $11 \times 12 \mathrm{deg}$ rectangle. All of these conditions produced about equal masking. The failure of any of these mask manipulations to affect the results suggests that the transient system, unlike the sustained system, is a single channel in the spatial frequency domain (Legge, 1978). Moreover, there appears to be little or no orientation tuning. Below, I will cite evidence from other studies which support these conclusions.

\section{Motion Aftereffects in Uniform Flicker}

The fact that flicker had no effect on motion perception with fine test gratings suggested that there might be two distinct mechanisms for detecting motion-one sensitive only below $4 \mathrm{c} / \mathrm{deg}$ and the other tuned to higher spatial frequencies (or low velocities). This view is consistent with the results of another experiment (Green et al., 1983), in which observers were tested for a motion aftereffect (MAE). The observers adapted to drifting gratings and then viewed one of two test fields, a stationary grating or uniform flicker. The stationary grating produced the typical MAE, which appeared as a slow drift of the test grating in the direction opposite that of adaptation. A quite different MAE was seen in the uniform flicker. It is difficult to describe except to say that it appeared as motion without distinct form. Most observers reported it as rapidly moving mist or fog. In addition to differences in appearance, the two aftereffects also differed in other respects. Unlike the typical pattern MAE, the flicker MAE could be obtained with adaptation only to spatial frequencies below $4 \mathrm{c} / \mathrm{deg}$ (Figure 4f).

Since the relationship between adaptation and test patterns in the MAE is so poorly understood, caution should be taken in interpreting this experiment. However, I am tempted to suggest that the transient system is responsible for the flicker MAE, since it is perceived as motion without distinct form, and the normal MAE may be thought of as an aftereffect of the sustained system. Whether or not this is true, it does appear that there is more than one direction-selective mechanism in human vision. The view that human 
vision contains at least two motion-detection mechanisms has been stated and restated numerous times since Exner (1875) first suggested the possibility (see, e.g., Bonnet, 1977; Leibowitz, 1955; Lovegrove, Over, \& Broerse, 1979; Pantle \& Picciano, 1976).

Two recent studies provide more evidence that motion is differentially processed above and below $3 \mathrm{c} / \mathrm{deg}$. R. DeValois (personal communication, 1981) had observers view gratings which rapidly oscillated back and forth. The amplitude of the excursion was adjusted until motion was barely perceptible. Below $3 \mathrm{c} / \mathrm{deg}$, this occurred at a fixed phase angle for all spatial frequencies. For finer gratings, however, motion was seen at a fixed distance of movement (see also Westheimer, 1978). This suggests that the one system processes phase (Green \& Blake, 1981), whereas the other depends on velocity. Watson and Robson (1981) compared thresholds of detection and spatial frequency discrimination for drifting gratings. Detection-discrimination ratios exhibited evidence of differences in temporal mechanisms above and below $2 \mathrm{c} / \mathrm{deg}$.

There is one additional reason to doubt that motion versus form thresholds can be used to distinguish sustained from transient mechanisms. When gratings are moved at a higher temporal rate, it is possible to see motion at absolute thresholds for increasingly fine gratings (Green, 1983a, 1983b; Lennie, 1980b; Watson, Thompson, Murphy, \& Nachmias, 1980). Therefore, the transient system should mediate detection at increasing spatial frequencies when faster temporal rates are employed. However, the 3-4-c/deg breakpoint between sustained and transient mechanisms does not seem to depend on temporal frequency. Neither differences in sensitivity to flicker nor differences in orientation tuning (Burbeck \& Kelly, 1982, discussed below) are affected by temporal frequency. This is not what would be expected if sustained and transient mechanisms were distinguishable by a perceived motion criterion. As gratings were moved faster, motion would be seen at the detection threshold of increasingly higher spatial frequencies. Therefore, the transient system would be mediating for detection at higher spatial frequencies.

\section{Relative Sensitivities to Form and Motion}

The results of the masking-by-light experiments suggest that the dichotomy between sustained and transient does not equal a dichotomy between pattern and motion/flicker analysis. As noted above, the sustained and transient mechanisms are presumed to differ not only in their roles in perception, but also on their spatiotemporal tuning. On the basis of subjective motion versus form thresholds, three such differences have been proposed: (1) The transient system is presumed to be tuned to lower spatial frequencies (Kulikowski \& Tolhurst, 1973; Tolhurst, 1973); (2) the sustained system is relatively more sen- sitive in central vision, whereas the transient system dominates the periphery (Breitmeyer \& Ganz, 1976; Sharpe, 1974); and (3) the sustained system exhibits an oblique effect, whereas the transient system shows little (Essock \& Lehmkuhle, 1982) or no (Camisa et al., 1977) anisotropy. I have tested (Green, 1983a) each of these proposed differences between sustained and transient mechanisms by means of the detectiondiscrimination paradigm.

In one experiment, observers performed detection discrimination on gratings of 28 combinations of spatial and temporal frequency. The motion parameter can be expressed in terms of temporal frequency (as is more often done) or velocity (temporal frequency/spatial frequency). When the detection-discrimination ratios were plotted on a temporal frequency axis, the curves for different spatial frequencies were parallel but displaced from one another by 1 octave. When plotted on a velocity axis, as shown in Figure 7, the curves for different spatial frequencies superimposed to produce a single function. The conclusion here is that equal velocities produce equal ratios: relative sensitivity to form and motion depends only on velocity. The special sensitivity for motion at low spatial frequencies results from the practice of maintaining constant temporal frequency as the spatial frequency is increased. (See also Harris, 1980, for a similar conclusion.) This result may seem to conflict with the DeValois study (referred to in the previous section), but he used relatively fast motion, and I used relatively slow motion. As others have found (e.g., Burbeck \& Kelly, 1982), the transient system is not stimulated at very slow motion rates. It seems likely that my study investigated only a sustained motion analyzer. Note also than when gratings were masked by light, the same curves were obtained

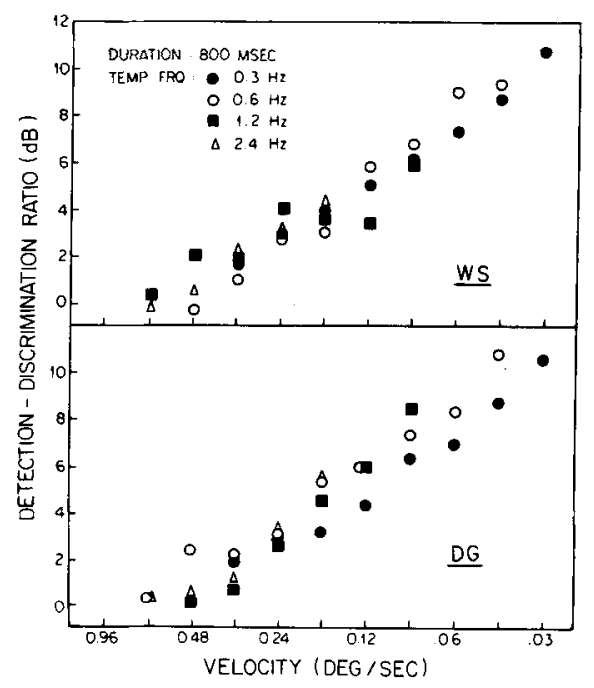

Figure 7. Ratio of detection to discrimination as a function of velocity of drifting gratings. Top and bottom pasel represent data for two differeat observers. 
whether either temporal frequency or velocity was held constant.

Ratios of detection and discrimination were also measured at different retinal eccentricities in order to see if the transient system increased relative sensitivity in the periphery. According to the sustainedtransient model, the ratio should have fallen in the periphery as the transient system gained relative sensitivity. The results, however, were just the opposite: the ratio rose, indicating a relative loss of motion sensitivity.

In the final test, observers viewed vertical and oblique gratings. Both detection and discrimination sensitivities were better with the vertical gratings. Moreover, the speed of the gratings had no effect on the magnitude of the retinal anisotropies. I found no evidence to support the claim that motion and form processing exhibit different degrees of retinal anisotropy. Moreover, Kelly (1975) has reported an oblique effect in gratings flicker as fast as $50 \mathrm{~Hz}$, and Schwartz, Winstead, and May (1982) have further found that another kind of temporal judgment, 2-pulse resolution, also exhibits an oblique effect at high spatial frequencies.

This set of experiments seems to be a major blow to any attempt to validate the sustained-transient dichotomy by comparing motion and form thresholds. At this point, there are two courses. First, the sustained-transient dichotomy could be totally dismissed. However, the masking-by-light studies, as well as others cited below, suggest that there is some basis for a sustained-transient dichotomy. The second possibility is to modify and update the model. In the next section, I will propose some criteria for distinguishing the two systems and cite converging evidence from other studies. The final section will outline a receptive field model that can account for most of these differences.

\section{PROPERTIES OF SUSTAINED AND TRANSIENT MECHANISMS}

The major criterion previously used (Keesey, 1972; Kulikowski \& Tolhurst, 1973) to distinguish sustained from transient systems was perception of pattern versus motion/flicker. Evidence presented above suggests that both mechanisms are capable of mediating motion perception. Likewise, several authors (Burbeck, 1981; Derrington \& Henning, 1981; Lennie, 1980b) ${ }^{2}$ have proposed that the transient system can signal at least some pattern information. Therefore, the previous basis for distinguishing the two systems seems invalid. Two secondary criteria, magnitude of the oblique effect and relative sensitivity in the central and peripheral fields, also fail if the motion/no-motion distinction is accepted.

There is also evidence to show that sustained and transient systems cannot be distinguished by the central-peripheral retina criterion. As noted above, variation of detection-discrimination ratio across the retina shows no gain of motion sensitivity in the periphery. In a particularly telling experiment, Wright and Johnson (1983) measured contrast sensitivity for gratings at different retinal eccentricities. Sensitivity for stationary and flickering gratings declined by the same amount as the target was imaged further in the periphery. If the transient system dominates the periphery, then flickering gratings should become more detectable than stationary ones. Virsu, Rovamo, Laurinen, and Nasanen (1982) performed a similar experiment and likewise concluded that, after scaling for cortical magnification, there was no qualitative difference in spatiotemporal vision between central and peripheral retina.

In order to retain a sustained-transient dichotomy, new distinguishing properties are needed. Table 1 lists a number of candidates, some of which have previously been suggested and still appear valid.

\section{Sensitivity to Uniform Flashes of Light}

Above, I have cited evidence from several of my own studies to suggest that uniform flicker desensitizes transient but not sustained mechanisms. Similar results have been reported by other authors. Klein et al. (1977) found that uniform flicker masked low spatial flickering gratings but not stationary ones, provided that care had been taken to switch the target gradually on and off. Stromeyer, Zeevi, and Klein (1979) reported that unpatterned flashes greatly masked broad flickering bars but had much less effect on narrow bars, which contain higher spatial frequencies. In an experiment similar to my flicker masking study, Breitmeyer, Levi, and Harwerth (1981) measured contrast sensitivity for flickering gratings in the presence of a steady and uniform flickering background. Although one observer showed data similar to mine, the other exhibited a masking effect at spatial frequencies above $4 \mathrm{c} / \mathrm{deg}$. I think that the difference in results may be traced to psychophysical methods. While I have used a forced-choice paradigm, Breitmeyer et al. employed the subjective method of adjustment. Trying to set a subjective cri-

Table 1

Candidates for Distinguishing Properties of a Sustained-Transient Dichotomy

\begin{tabular}{lll}
\multicolumn{1}{c}{$\begin{array}{c}\text { Transient } \\
\text { System }\end{array}$} & \multicolumn{1}{c}{ Property } & \multicolumn{1}{c}{$\begin{array}{c}\text { Sustained } \\
\text { System }\end{array}$} \\
\hline Yes & Masked by Uniform Flashes & No \\
Fast & Temporal Summation & Slow \\
Fast & Latency of Response & Slow \\
Broad & Orientation Tuning & Narrow \\
Larger & Binocular Summation & Smaller \\
$0-4 \mathrm{c} / \mathrm{deg}$ & Spatial Frequency Range & $?-60 \mathrm{c} / \mathrm{deg}$ \\
One or Many & Number of Spatial Channels & Many \\
Several & Number of Temporal Channels & One? \\
\hline
\end{tabular}


terion in a flickering masking experiment would be extremely difficult. However, when Breitmeyer et al. measured reaction times to suprathreshold gratings, flicker had an effect only when spatial frequencies were $2 \mathrm{c} / \mathrm{deg}$ or below. This result is consistent with a number of other reaction time studies (see below).

A final piece of evidence originates in a study by Braddick (1973), which employed a masking by light situation. Observers viewed two sequentially presented "frames," each consisting of random dots with no obvious contours or pattern. If flashed with a short dark ISI, the observer perceived coherent motion of a correlated submatrix within the frames. A uniform flash presented between frames, however, abolished the percept. Braddick also employed two other frames, each of which contained a form that could easily be perceived without sequential presentation. As before, flashing one frame after the other produced apparent coherent motion. In this instance, however, a uniform flash had no effect on perceived motion. Presumably, uniform flashes masked the transient mechanisms that were responsible for detecting motion. Once form had been segregated from the background by the sustained system, uniform flashes produced no masking.

\section{Temporal Summation}

Several studies (Breitmeyer \& Ganz, 1977; Brown \& Black, 1976; Legge, 1978) have found that the period of temporal summation is faster for low than for high spatial frequency gratings. This fact by itself does not particularly support the dichotomy, because the lengthening of the critical period is gradual as spatial frequency increases and there is no discontinuity indicative of two separate mechanisms. However, Legge (1978) further measured temporal summation with the on- and offset of the target transiently masked. This procedure elevated threshold and slowed summation for detection of grating below $3 \mathrm{c} / \mathrm{deg}$, a range consistent with the effects of uniform flicker masking.

\section{Latency of Response}

It has been reported in a number of studies that reaction time is longer to high than to low spatial frequency gratings. As noted above, this by itself does not provide convincing support for the existence of two mechanisms. Neither Breitmeyer (1975) nor Lupp et al. (1976) found any discontinuities in the reaction with variation in spatial frequency. However, Vassilev and Mitrov (1976) reported that reaction times were similar for all spatial frequencies at and below $4 \mathrm{c} / \mathrm{deg}$. Only above this point did reaction time increase with spatial frequency. A finegrain analysis of reaction times has been used to uncover stronger evidence for a relationship between latency and spatial frequency. Harwerth and Levi (1978) measured reaction times as a function of con- trast for different spatial frequencies. They concluded that the curves showed breaks which indicated a switch from sustained to transient mechanisms. However, the break points in the fitted curves seem rather arbitrary and not overly convincing. Stronger evidence for a dichotomy was reported by Tolhurst (1975), who measured reaction times to $0.2-$ and $3.5-\mathrm{c} / \mathrm{deg}$ gratings. He found that the reaction time distribution to the lower spatial frequency was bimodal. Each mode was associated with an on- and offset transient of the grating. On the other hand, the 3.5$\mathrm{c} /$ deg grating produced a unimodal distribution that was not affected by whether transients were present or absent. Presumably, the observer detected the onand offset of the coarse grating with the transient system but used the sustained system to detect the finer grating. Further evidence for a division in reaction time mechanisms at about $3 \mathrm{c} / \mathrm{deg}$ comes from Breitmeyer et al. (1981), who found that uniform flicker masks reduced reaction times for gratings with spatial frequencies only as high as $2 \mathrm{c} / \mathrm{deg}$.

\section{Orientation Tuning}

Much evidence suggests that while the sustained system has narrow orientation tuning, the transient system exhibits little or no meridional selectivity. In one of the flicker masking studies (Green, 1983b) cited above, it was found that changing the orientation of a flickering bar had little effect on the size of masking. A previous study (Sharpe \& Tolhurst, 1973) of orientation-specific adaptation has shown that flickering or drifting of low spatial frequency gratings can greatly expand the orientation bandwidth of the threshold elevation. The greatest evidence for a difference in orientation selectivity is found in studies of subthreshold summation. Kulikowski, Abadi, and King-Smith (1973) found that there was little or no summation between steadily presented gratings if their orientations differed by more than 3-6 deg. In contrast, two other studies (Burbeck \& Kelly, 1982; Gorea, 1979) have reported almost complete summation between low spatial frequency flickering gratings that were oriented orthogonally. The more extensive study by Burbeck and Kelly employed gratings over a very wide range of spatial and temporal frequencies. With $0-$ or $1-\mathrm{Hz}$ flicker, little summation was found at any spatial frequency. When temporal rate was increased up to a maximum of $30 \mathrm{~Hz}$, however, a high degree of summation was found with gratings as fine as $2 \mathrm{c} / \mathrm{deg}$. At $4 \mathrm{c} / \mathrm{deg}$ and higher, there was little summation at any flicker rate, even though the observers could surely see the $30-\mathrm{Hz}$ flicker at all spatial frequencies. What additivity that was found may have been due to probability summation rather than a neural summation. The fact that the break point between the two mechanisms was (1) at $2-4 \mathrm{c} / \mathrm{deg}$ and (2) not affected by flicker rate above $1 \mathrm{~Hz}$ is consistent with 
the flicker masking data. As noted above, the upper limit of flicker masking was $2-4 \mathrm{c} / \mathrm{deg}$ over a range of temporal frequencies from 2.2 to $16 \mathrm{~Hz}$. A final piece of evidence on orientation tuning comes from a study of induced tilt by Matin (1974). In a variant of the Crawford (1947) paradigm, Matin briefly presented test gratings at various times before, during, or after a 3-sec inducing grating of different orientation. The apparent tilt produced by the inducing grating was maximal at the on- and offset, creating "ears" similar to those found in standard Crawford experiments. The huge size of the transiently induced tilt, 20-25 deg, is consistent with a relatively broad orientation tuning for transient mechanisms.

\section{Binocular Summation}

In an early study, Campbell and Green (1965) compared grating contrast sensitivity under conditions in which the observer viewed a stationary target with one or both eyes. Binocular viewing produced sensitivity that was $1.4(\sqrt{2})$ times higher than that found with monocular viewing, a result subsequently confirmed in a number of other studies (see a review by Blake \& Fox, 1973). Several more recent studies, however, have reported that flicker gratings produce significantly more binocular summation. Rose (1978) found $80 \%$ to $90 \%$ summation in the detection of flickering gratings, and Cavonius (1979) reported $80 \%$ summation for flickering spots. Although finding somewhat less summation, in the $60 \%-70 \%$ range, Blake and Rush (1980) also found higher than $\sqrt{2}$ summation with flickering gratings. Other kinds of binocular interaction seem to be stronger for transient mechanisms. For example, the luminance of a uniform field presented to one eye may alter sensitivity to flickering stimuli presented to the other (Lipkin, 1962). On the other hand, luminance of a uniform field has no effect on contrast sensitivity for detecting stationary gratings presented to the other eye (Blake, Breitmeyer, \& Green, 1980).

\section{Spatial Frequency Range}

The results of flicker masking suggest that the transient system operates only at spatial frequencies as high as $2-4 \mathrm{c} / \mathrm{deg}$. This $2-4-\mathrm{c} / \mathrm{deg}$ upper limit is supported by a number of different kinds of studies, some of which have been cited above. The major evidence is: (1) flicker masking of contrast thresholds, (2) flicker masking of reaction time (Breitmeyer et al., 1981), (3) motion aftereffects in uniform flickering fields (Green et al., 1983), (4) reaction times to gratings of different spatial frequency (Tolhurst, 1975; Vassilev \& Mitrov, 1976, (5) spatial frequency masking (Legge, 1978), (6) comparison of on-off and flickering gratings (Kulikowski, 1971). (7) subthreshold summation of aperiodic stimuli (Wilson, 1980), and (8) subthreshold summation of orthogonal gratings (Burbeck \& Kelly, 1982). Two other studies also report similar data. Breitmeyer and Julesz (1975) found that sharp, rather than gradual, onset improves grating sensitivity only as high as $5 \mathrm{c} / \mathrm{deg}$. Wilson and Bergen (1979) derived two distinct transient mechanisms from observers' contrast sensitivities. One mechanism had an upper cutoff of $4 \mathrm{c} / \mathrm{deg}$, whereas the other extended to as high as $7-8 \mathrm{c} / \mathrm{deg}$.

The sum of these studies are consistent with a model proposed by Legge (1978) and shown in Figure 8 . He views the transient system as a single lowpass filter with an upper limit of about $4 \mathrm{c} / \mathrm{deg}$. The sustained system operates from as low as $0.375 \mathrm{c} / \mathrm{deg}$ to higher spatial frequencies, with the upper cutoff probably being the acuity limit of about $60 \mathrm{c} / \mathrm{deg}$. [A study by Stromeyer, Klein, Dawson, \& Spillman (1982) suggests that the sustained system may extend even to low spatial frequencies.] In Legge's experiment, detection was mediated by the transient system up to a spatial frequency as high as $1.5 \mathrm{c} / \mathrm{deg}$, where the sensitivity profiles of the two systems cross. A transient mask selectively depressed the transient system so the sustained system could mediate detection at lower spatial frequencies. His result is complementary to one cited above (Green, 1981b): prior adaptation to flicker raises detection thresholds for 8-Hz on-off flickering gratings only when spatial frequencies are as high as $1.5 \mathrm{c} / \mathrm{deg}$. When subjects used a flicker criterion for setting threshold, the adaptation effect increased to $4 \mathrm{c} / \mathrm{deg}$.

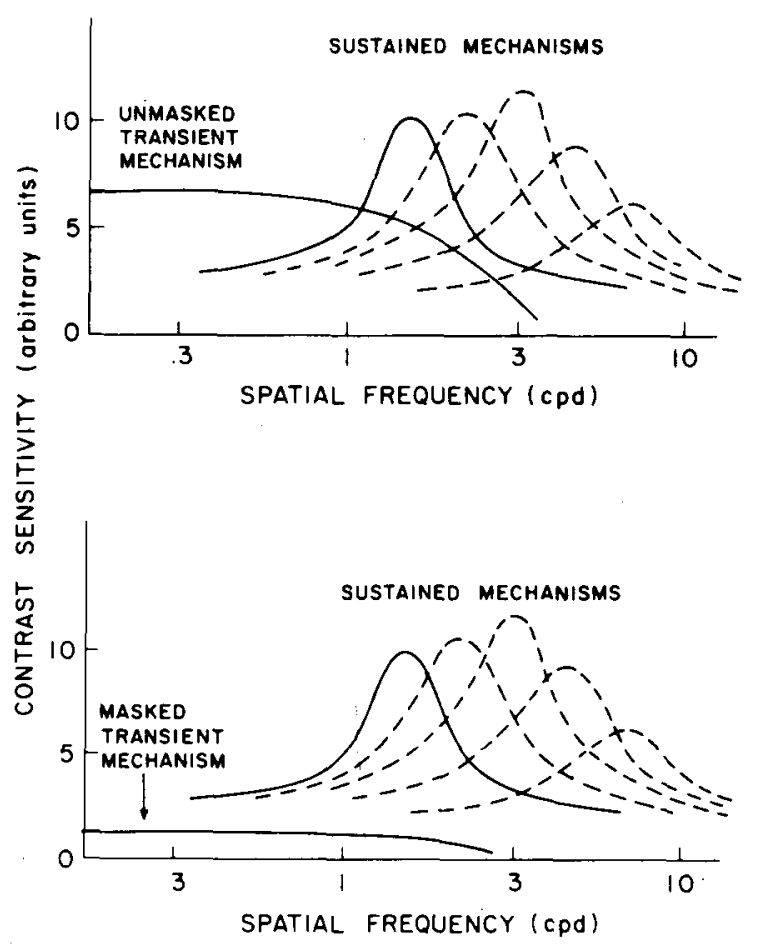

Figure 8. Hypothetical tuning of sustained and transient mechanisms suggested by Legge (1978). 
The assertion that the transient system operates only as high as $4 \mathrm{c} / \mathrm{deg}$ is in conflict with the original version of the dichotomy. Kulikowski and Tolhurst (1973) indicate that the transient system extends to at least $20 \mathrm{c} / \mathrm{deg}$, the highest used in their study. A more recent paper (Murray, MacCana, \& Kulikowski, 1983) shows upper limits ranging from 20 to $28 \mathrm{c} / \mathrm{deg}$, depending on temporal factors. As in most studies, the conclusion that the transient system has sensitivity at high spatial frequencies depended entirely on subjective pattern and motion thresholds. In my view, this is not a tenable criterion, since both systems may signal motion.

\section{Number of Spatial Frequency Channels}

Manipulating the size of the flicker mask had no effect on the magnitude of masking or on the spatial frequency range of the gratings from which a threshold elevation could be obtained. A possible conclusion to be drawn is that the transient system is a single low-pass spatial frequency channel, as suggested by Legge's (1978) diagram (see also Wilson, 1980). This would, of course, contrast with the sustained system, which generally behaves as a multiple-channel system (Blakemore \& Campbell, 1969). However, other data suggest that the transient system can behave as a multiple-channel system. Graham (1972) and Stromeyer et al. (1982) compared the bandwidth of spatial frequency channels for stationary and drifting gratings in a selective adaptation paradigm. Bandwidths obtained with temporally modulated gratings were broader than those obtained with steady gratings. Likewise, Pantle (1973) reported that the bandwidth of subthreshold summation was greater for rapidly drifting gratings, but only for spatial frequencies below $5 \mathrm{c} / \mathrm{deg}$. On the other hand, Arend and Lange (1979) found no effect of exposure duration on channel width. The reason why the transient system may behave as a single channel in some situations and as a multiple channel in others will be examined in the discussions, below, of receptive field properties.

\section{Number of Temporal Channels}

Several studies have examined the possible existence of temporal channels in adaptation paradigms. When (1) adapting and testing with uniform flicker (Smith, 1970, 1971; Nilsson, Richmond, \& Nelson, 1975), (2) adapting and testing with drifting gratings of different velocity (Pantle \& Sekuler, 1968), and (3) adapting to flicker and testing with motion (Green, 1981b), it has been found that broad temporal channels exist. However, these channels are found at temporal frequencies only at and above about $6 \mathrm{~Hz}$. No study has found evidence for temporal channels centered at low rates. Since it is likely that slow temporal modulation is processed by the sustained system (Burbeck \& Kelly, 1982), this suggests that the sustained system is a single temporal channel, whereas the transient system contains multiple (albeit broad) temporal channels. All of the temporal tuning studies have been performed with low spatial frequency gratings. The prediction based on my reasoning would be that no temporal channels would be found if gratings above $4 \mathrm{c} / \mathrm{deg}$ were used. This remains to be tested.

\section{SUSTAINED AND TRANSIENT RECEPTIVE FIELDS}

The view that uniform flicker masks only the transient system provides insight as to the receptive field properties of the two mechanisms. What kinds of receptive fields would or would not respond to uniform flashes? Receptive fields are determined by the interactions of two forces, excitation and inhibition. Most authors have been concerned primarily with the spatial distribution of these two influences. A prototypical receptive field consists of an excitatory area surrounded or flanked by inhibitory regions (or the reverse). When a uniform flash covers the entire receptive field, all of the excitatory and inhibitory areas are stimulated. (This is similar to what happens when the "null" position of a cell is found, where excitatory and inhibitory areas are equally stimulated.) No net response would occur if (1) the excitatory and inhibitory areas were equal in strength, and (2) the time course of excitation and inhibition were identical. Violation of either of these conditions would allow uniform flicker to produce a response. Psychophysical evidence seems to suggest that inhibition in transient mechanisms is delayed relative to excitation. For example, one method of determining the time course of inhibition is to measure temporal frequency response functions. Mechanisms with an asynchrony between excitation and inhibition would exhibit temporal frequency responses very different from those in which excitation develops simultaneously. A mechanism with inhibition delayed relative to excitation (or vice versa) would produce a bandpass temporal frequency response. With no delayed inhibition, temporal tuning curves would exhibit a low-pass shape. This is consistent with the usual view of the sustainedtransient dichotomy (Figure 1). At least some authors (Robson, 1966; Koenderink \& van Doorn, 1979) have found bandpass curves for sine gratings with spatial frequencies below $4 \mathrm{c} / \mathrm{deg}$, but low-pass curves at higher spatial frequencies.

The temporal response properties of mechanisms tuned to different spatial frequencies have also been investigated by Watson and Nachmias (1977), who measured sensitivity to double pulses of gratings. The results showed that only low spatial frequency mechanisms responded with delayed inhibition. This result is especially impressive because their very brief $4 \mathrm{msec}$ exposure duration strongly favors the transient system due to its briefer summation period (Brown \& 
Black, 1976; Breitmeyer \& Ganz, 1977; Legge, 1978). It therefore seems that higher spatial frequency mechanisms will not exhibit delayed inhibition under any conditions. As noted earlier, neither the flicker masking data nor the summation experiments of Burbeck and Kelly (1982) found any evidence that increasing temporal frequency could push the transientsustained border above $4 \mathrm{c} / \mathrm{deg}$. In the case of Watson and Nachmias, delayed inhibition was found at $3.5 \mathrm{c} / \mathrm{deg}$ but very little was found at $7.0 \mathrm{c} / \mathrm{deg}$.

The presence or absence of delayed inhibitions can also explain why the two mechanisms produce somewhat different percepts. Ratliff (1965) has pointed out that the inhibitory spatial side bands of receptive fields perform a mathematical function of differentiation. This serves to enhance the sharpness of the neural image and thereby improve spatial resolution. It is equally true that delaying inhibition will produce luminance differentiation in time and promote good temporal resolution. However, delaying inhibition will reduce the amount of spatial differentiation. Figure 9 shows hypothetical sustained and transient receptive fields. The sustained field is characterized by spatially distributed inhibition, in order to produce optimal spatial acuity. In the transient receptive field, the inhibition is delayed to increase the temporal resolution. If the assumption is made that total inhibition is roughly constant, then this is accomplished at the expense of reducing the spatial sidebands. That is, a slice through the time axis would reveal that the inhibitory spatial side bands were reduced or entirely eliminated if the delay was great enough (see Wilson \& Bergen, 1979, Figure 9). As a result there is a tradeoff between spatial and temporal distribution of inhibition: the sustained mechanisms can produce good spatial acuity but not good temporal resolution, whereas the transient system can produce specialized temporal resolution.
From this scheme, it becomes clear why the transient system behaves as a single channel in some cases and as a multiple channel in others. Stimulation by unpatterned flashes will activate any mechanisms that respond with delayed inhibition. Adaptation and masking by light studies therefore produce singlechannel results with a low-pass tuning. Multiplechannel results can also be obtained if the inhibition and excitation are asynchronous but still overlap in time. This will result in the inhibitory spatial sidebands' being reduced but not eliminated. Rather than being entirely low pass, the tuning would broaden on the low spatial frequency side of the band. Graham's (1972) experiment was designed to look for exactly this effect. She found that some slight broadening occurred with moving gratings, the larger effect being on the low-frequency side of the band. Magnitude of the broadening was much less than expected, but the relatively small size of the expansion may have been due to (1) the fact that the lowest test frequency employed was only $1.4 \mathrm{c} / \mathrm{deg}$, which is just getting into the range where I would expect the effect to occur, and (2) the averaging of the results over low and high spatial frequencies. Stromeyer et al. (1982), on the other hand, employed gratings much coarser than those employed by Graham and found that transient stimuli produced a very large broadening of the low spatial frequency side of the channel. The increase in channel width, however, was not obtained with stationary gratings which were gradually switched on and off. In sum, the effect of temporally modulating gratings on the form of spatial frequency channels is consistent with the view that spatial sidebands are reduced due to partial delay of inhibition.

Above, I have suggested that the delay of inhibition and the resulting loss of sidebands can account for (1) the relative form- and motion-signaling ability

\section{SUSTAINED RECEPTIVE FIELD}
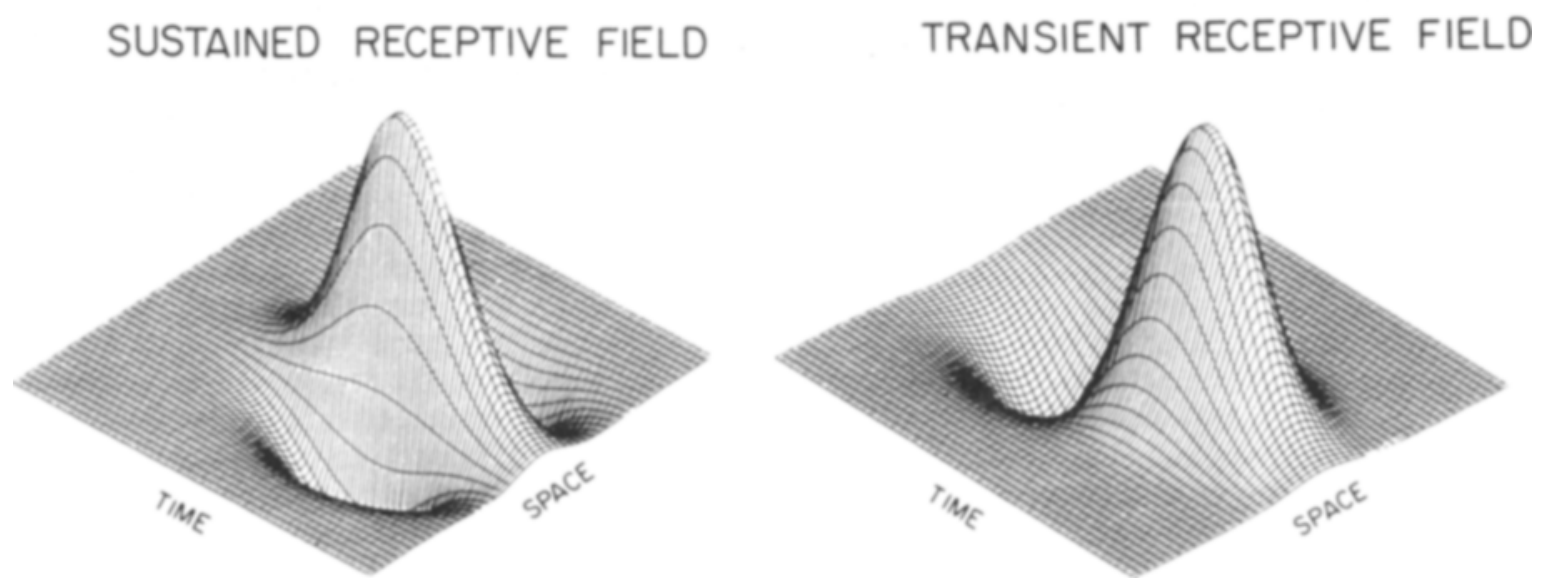

Figure 9. Hypothetical response properties of sustained and transient mechanisms. Points above the plane represent excitatory response while dips below signify inhibitory activity. 
of the sustained and transient mechanisms, (2) the fact that the transient system may behave as a single or multiple channel, and (3) the unique sensitivity of the transient system to uniform flashes. The model also accounts for the relatively poor orientation tuning of the transient system. Bars aligned far off the preferred orientation might still produce a net positive response if spatial inhibition were sufficiently weak due to the delay. Likewise, the presence of temporal channels in the transient, but not sustained, system could be explained by the delay of inhibition. The delayed negative component would produce a bandpass temporal response function. Different optimal frequencies would result from various asynchronies between excitation and inhibition. Without delayed inhibition, however, the sustained system would be low pass with no channels possible.

\section{CONCLUSION}

In this article, I have examined the question of whether the human visual system contains separate sustained and transient mechanisms. The bulk of the data suggests that the original formulation of the dichotomy is not supportable: there is little evidence to confirm that the visual system contains separate form- and motion-analyzing systems. Moreover, the putative differences in spatial frequency tuning, retinal anisotropy, and differential distribution in central and peripheral vision do not appear valid. However, data from many studies provide several other criteria by which the two mechanisms may be distinguished.

It might be best to think of the two mechanisms as being complementary in the sense that scotopic and photopic mechanisms are complementary. Photopic vision provides better form vision but has poor absolute sensitivity to light. The scotopic system, on the other hand, has good absolute sensitivity, but gains this by trading increased spatial summation against a relative loss of acuity. Both systems can signal something about brightness as well as something about form, but each performs a specialized role in order to extend the range of conditions over which vision may occur. In much the same way, sustained and transient mechanisms use the synchrony of excitation and inhibition to trade off between good spatial and good temporal resolution. As a result, the human visual system is capable of operating over a greater range of conditions.

\section{REFERENCES}

Arend, L., \& Lange, R. (1979). Influence of exposure duration on the tuning of spatial frequency channels. Vision Research, 19, $195-200$.

Barlow, H. (1972). Light and dark adaptation: Psychophysics. In L. Hurvich \& D. Jameson (Eds.), Handbook of sensory physiology (Vol. VII/4, pp. 1-27). New York: Springer-Verlag.
Blake, R., Breitmeyer, B., \& Green, M. (1980). Contrast sensitivity and binocular brightness: Dioptic and dichoptic conditions. Perception \& Psychophysics, 27, 180-181.

Blake, R., \& Fox, R. (1973). The psychophysical inquiry into binocular summation. Perception \& Psychophysics, 14, 161-185.

Blake, R., \& Rush, C. (1980). Temporal properties of binocular mechanisms in the human visual system. Experimental Brain Research, 38, 333-340.

Blakemore, C., \& Campeell, F. W. (1969). On the existence of neurons in the human visual system selectively sensitive to orientation and size of retinal images. Journal of Physiology (London), 203, 237-260.

Bonnet, C, (1977). Visual motion detection models: Features and frequency filters. Perception, 6, 491-500.

Bowditch, H., \& Hale, G. (1881). Optical illusion of motion. Journal of Physiology (London), 3, 296-301.

Bow KER, D., \& KEESEY, U. (1983). Sensitivity to countermodulating gratings following spatio-temporal modulation. Journal of the Optical Society of America, 73, 427-440.

Boynton, R. M. (1961). Some temporal factors in vision. In W. A. Rosenblith (Ed.), Sensory communication. New York: Wiley.

Braddick, O. (1973). The masking of apparent motion in randomdot patterns. Vision Research, 13, 355-369.

BRE ITMEYER, B. (1975). Simple reaction time as a measure of the temporal response properties of transient and sustained channels. Vision Research, 15, 1411-1412.

BRE ITMEYER, B., \& GANZ, L. (1976). Implications of sustained and transient channels for theories of visual pattern masking, saccadic suppression and information processing. Psychological Review, 83, 1-36.

Breitmeyer, B. G., \& Ganz, L. (1977). Temporal studies with flashed gratings: Inferences about human transient and sustained channels. Vision Research, 17, 861-866.

BrettmeYer, B., \& Julesz, B. (1975). The role of on and off transients in determining the psychophysical spatial frequency response. Vision Research, 15, 411-416.

Breitmeyer, B., Levi, D., \& Harwerth, R. (1981). Flicker masking in spatial vision. Vision Research, 21, 1377-1385.

Brown, J. L., \& Black, J. E. (1976). Critical duration for resolution of acuity targets. Vision Research, 16, 309-315.

Burbeck, C. (1981). Criterion-free pattern and flicker thresholds. Journal of the Optical Society of America, 71, 1343-1350.

Burbeck, C., \& KeLtY, D. (1982). Contrast gain measurements and the transient/sustained dichotomy. Journal of the Optical Society of America, 71, 1335-1342.

Camisa, J., Blake, R., \& Lema, S. (1977). The effects of temporal modulation on the oblique effect in humans. Perception, 6, 165-171.

Campbell, R., \& Green, D. (1965). Monocular vs. binocular visual acuity. Nature, $208,191-192$.

Cavonius, C. R. (1979). Binocular interactions in flicker. Quarterly Journal of Experimental Psychology, 23, 273-280.

Crawford, B. (1947). Visual adaptation relation to brief conditioning flashes. Proceedings of the Royal Society, London, 134B, 283-302.

Derrington, A., \& Hennina, G. (1981). Pattern discrimination with flickering stimuli. Vision Research, 21, \$97-602.

Enroth-Cugall, C., \& Robson, J. G. (1966). The contrast sensitivity of retinal ganglion cells of the cat. Journal of Physiology (London), 187, 517-552.

Essock, E., \& LeHMкuHLE, S. (1982). The oblique effects of pattern and flicker sensitivity: Implications for mixed physiological input. Perception, 11, 441-445.

ExNER, S. (1875). Über des Sehen von Bewegung und die Theorie des zusammengesetzten Auges. Sitzungsberichts Akademie Wissenschaft Wien, 72, 156-190.

Gore A, A. (1979). Directional and nondirectional coding of a spatiotemporal modulated stimulus. Vision Research, 19, 545-549.

Graham, N. (1972). Spatial frequency channels in the human vi- 
sual system: Effects of luminance and pattern drift rate. Vision Research, 12, 53-68.

Green, M. (1981a). Spatial frequency effects in masking by light. Vision Research, 18, 861-866.

Green, M. (1981b). Psychophysical relationships among mechanisms sensitive to pattern flicker and motion. Vision Research, 21, 971-984.

Green, M. (1983a). Contrast detection and direction discrimination of drifting gratings. Vision Research, 23, 281-289.

GREEN, M. (1983b). Detection and discrimination of drifting gratings masked by flicker. Manuscript submitted for publication.

Green, M. (1983c). Visual masking by flickering surrounds. Vision Research, 23, 735-744.

Green, M., \& Blake, R. (1981). Phase effects in monoptic and dichoptic temporal integration: Flicker and motion detection. Vision Research, 21, 365-372.

Green, M., Chilcoat, M., \& Stromeyer, C. (1983). Motion aftereffects in spatially uniform flicker fields. Nature, 304, 61-62.

Green, M., \& ODOM, J. V. (1984). Interocular transfer of masking by light. Perception \& Psychophysics, 35, 265-268.

HARRIs, M. (1980). Velocity specificity of the flicker to pattern sensitivity ratio in human vision. Vision Research, 20, 687-691.

Harwerth, R. S., \& Levi, D. M. (1978). Reaction time as a measure of suprathreshold grating detection. Vision Research, 18, 1579-1586.

KaHNEman, D. (1968). Method, findings and theory in studies of visual masking. Psychological Bulletin, 70, 404-425.

KEESEY, U. T. (1972). Flicker and pattern detection: A comparison of thresholds. Journal of the Optical Society of America, 62, 446-448.

KELLY, D. (1975). No oblique in the chromatic pathways. Journal of the Optical Society of America, 65, 1512-1514.

Kitterle, F. L., \& Leguire, L. E. (1975). The effects of borders and contour on threshold during early dark adaptation. Vision Research, 15, 1217-1224.

Kuein, S., Stromeyer, C., \& Madsen, J. (1977, April). Counterphase flickering and moving gratings masked with spatially uniform, flickering fields. Paper presented at the annual meeting of the Association for Research in Vision and Ophthalmology, Sarasota.

Koenderink, J., \& van Doorn, A. (1979). Spatiotemporal contrast detection threshold surface is bimodal. Optics Letters, 8 , 32-34.

KULIKowsKI, J. J. (1971). Some parameters affecting spatial and temporal resolution in humans. Vision Research, 11, 88-93.

Kulikowski, J., Abadi, R., \& King-Smith, P. (1973). Orientational selectivity of gratings and line detectors in human vision. Vision Research, 13, 1479-1486.

Kulikowski, J. J., \& Tolmurst, D. J. (1973). Psychophysical evidence for sustained and transient detectors in human vision. Journal of Physiology (London), 232, 149-162.

LEGGE, G. (1978). Sustained and transient mechanisms in human vision: Temporal and spatial properties. Vision Research, 18, 69-82.

LE iBowitz, H. (1955). The relation between the rate threshold for the perception of movement and luminance for various durations of exposure. Journal of Experimental Psychology, 49, 209-214.

Lennie, P. (1980a). Parallel visual pathways: A review. Vision Research, 20, 561-594.

Lennie, P. (1980b). Perceptual signs of parallel pathways. Philosophical Transactions of the Royal Society of London, 290B, 23-37.

Levelt, W. (1968). On binocular rivalry. Soesterberg, The Netherlands: Institute for Perception RVO-TNO.

LEVI, D., \& HARWEnTH, R. (1977). Spatio-temporal interactions in anisometric and strabismic amblyopia. Investigative Ophthalmology and Visual Science, 16, 90-95.

Levinson, E., \& Sexuler, R. (1975). The independence of channels in human vision selective for direction of movement. Jour nal of Physiology (London), 250, 347-366.
LIPKIN, B. (1962). Monocular flicker discrimination as $\mathbf{a}$ function of area and luminance of contralateral steady light. Journal of the Optical Society of America, 52, 1287-1295.

Lovegrove, W., Over, W., \& Broerse, J. (1979). Color selectivity in motion aftereffect. Perception \& Psychophysics, 25, 157-159.

Lupp, U., Hauske, G., \& Wolf, W. (1976). Perceptual latency to sinusoidal gratings. Vision Research, 16, 969-972.

Matin, E. (1974). Light adaptation and the dynamics of induced tilt. Vision Research, 14, 255-265.

Murray, I., MacCana, F., \& Kulikowski, J. J. (1983). Contribution of two movement detecting mechanisms to central and peripheral vision. Vision Research, 23, 151-159.

van Nes, F. L., Koenderink, J. J., Nas, H., \& Bouman, M. A. (1967). Spatiotemporal modulation transfer function of the human eye. Journal of the Optical Society of America, 57, 1082-1088.

Nilsson, T., Richmond, C., \& Nelson, T. (1975). Flicker adaptation shows evidence of many channels selectively sensitive to temporal frequency. Vision Research, 15, 621-623.

Pantle, A. (1973). Visual effects of sinusoidal components of complex gratings: Independent or additive? Vision Research, 13, 2195-2204.

Pantle, A. J. (1983). Temporal determinants of spatial sine-wave masking. Vision Research, 23, 749-757.

Pantle, A. J., \& Picciano, L. (1976). A multistable movement display: Evidence for two separate motion systems in human vision. Science, 193, 500-502.

Pantle, A., \& Sekuler, R. (1968). Velocity-sensitive elements in human vision: Initial psychophysical evidence. Vision $R e$ search, $8,445-450$.

Patel, A. S. (1966). Spatial resolution in the human visual system: Effect of mean retinal illuminance. Journal of the Optical Society of America, 56, 689-694.

RATLifF, F. (1965). Mach bands: Quantitative studies on neural networks in the retina. San Francisco: Holden-Day.

RoBSon, J. (1966). Spatial and temporal contrast sensitivity functions of the eye. Journal of the Optical Society of America, 56, 1141 .

Rose, D. (1978). Monocular vs. binocular contrast thresholds for movement and pattern. Perception, 7, 195-200.

Schiller, P. (1965). Monoptic and dichoptic visual masking by pattern and flicker. Journal of Experimental Psychology, 69, 193-199.

Schwartz, B., Winstead, D., \& MaY, J. (1982). Meridional differences in temporal resolution. Perception, 11, 25-34.

SHARPE, C. (1974). The contrast sensitivity of the peripheral visual field to drifting sinusoidal gratings. Vision Research, 14, 905-906.

Sharpe, C., \& Tolmurst, D. (1973). The effects of temporal modulation on the orientation channels of the human visual system. Perception, 2, 23-29.

Sмiтh, R. A. (1970). Adaptation of visual contrast sensitivity to specific temporal frequencies. Vision Research, 10, 275-279.

Smith, R. A. (1971). Studies of temporal frequency adaptation in visual contrast sensitivity. Journal of Physiology (London), 216, 531-552.

SPe RLiNG, G. (1965). Temporal and spatial masking. I. Masking by impulse flashes. Journal of the Optical Society of America, 55, $541-559$.

Stromeye R, C., \& Julesz, B. (1972). Spatial frequency masking in vision: Critical bands and spread of masking. Journal of the Optical Society of America, 62, 1221-1232.

Stromeyer, C., Klein, S., Dawson, B., \& Spillman, L. (1982). Low spatial-frequency channels in human vision: Adaptation and masking. Vision Research, 22, 225-234.

Stromeyer, C., Zeevi, Y., \& Klein, S. (1979). Response of visual mechanisms to stimulus onsets and offsets. Journal of the Optical Society of America, 69, 1350-1354.

Tolnurst, D. J. (1973). Separate channels for the analysis of shape and movement of a moving visual stimulus. Journal of Physiology (London), 231, 385-402. 
Tolhurst, D. (1975). Reaction times in the detection of gratings by human observers: A probabilistic mechanism. Vision Research, 15, 1143-1149.

Vassilev, A., \& Mitrov, D. (1976). Perception time and spatial frequency. Vision Research, 16, 89-92.

Virsu, V., Rovamo, J., Leurinen, P., \& Nasanen, R. (1982). Temporal contrast sensitivity, and cortical magnification. $\mathrm{Vi}$ sion Research, 22, 1211-1217.

Wagman, I., \& BatTersby, W. (1959). Neural limitations of visual excitability. II: Retrochiasmal interaction. American Journal of Physiology, 197, 1237-1242.

Watson, A. B., \& Nachmias, J. (1977). Patterns of temporal interaction in the detection of gratings. Vision Research, 17, 893-902.

Watson, A. B., \& RoBson, J. (1981). Discrimination at threshold: Labelled detectors in human vision. Vision Research, 22, 11151122.

Watson, A., Thompson, P., Munphy, B., \& Nachmias, J. (1980). Summation and discrimination of gratings moving in opposite directions. Vision Research, 20, 341-347.

Westheimer, G. (1978). Spatial phase sensitivity for sinusoidal grating targets. Vision Research, 18, 1073-1074.

WILSON, H. (1980). Spatiotemporal characterization of a transient mechanism in the human visual system. Vision Research, 20, 443-452.

Wilson, H., \& Bergen, J. (1979). A four mechanism model for threshold spatial vision. Vision Research, 19, 19-32.

Wright, M., \& Johnson, A. (1983). Spatiotemporal contrast sensitivity and visual field locus. Vision Research, 23, 983-989.

\section{NOTES}

1. Bowker and Keesey (1983) reported obtaining a flicker adaptation effect only after some difficulty. The reason undoubtedly was the length of his test intervals. Each was $3.8 \mathrm{sec}$ with the adaptation pattern switched on and off in a gradual 900 -msec envelope. The flicker adaptation effect is very transient and disappears entirely within a few seconds (Green, Chilcoat, \& Stromeyer, 1983), so brief test periods are necessary to produce a large ef fect. I eventually switched from adaptation to masking in order to avoid this problem. Bowker also claims that the effect is obtained only with a small field size. I have obtained flicker adaptation with $100-\mathrm{deg}$ wide fields (Green, 1981 b) when test periods were brief.

2. However, both Burbeck (1981) and Derrington and Henning (1981) overlook an alternative interpretation of their data. In both studies, observers discriminated orientation of vertical and horizontal flickering gratings. Since observers could perform this task at detection threshold, it was assumed that spatial information was available. However, previous studies (e.g., Levinson \& Sekuler, 1975) have shown that a counterphase flickering grating is detected by units tuned to one of the opposite directions of motion. Therefore, observers may actually have been discriminating left/right (vertical grating) from up/down (horizontal grating) motion. Using the latter explanation, one need not assume the existence of form information in transient mechanisms.

(Manuscript received December 9, 1983; revision accepted for publication March 28, 1984.) 